\title{
Evaluation of Skin Tissue Effects of Two-Pass Helium Plasma Treatment in Comparison to Nitrogen Plasma Using a Swine Model
}

\author{
Paul G Ruff IV* \\ West End Plastic Surgery Center, Washington, DC, USA \\ *Corresponding author: Paul G Ruff IV, West End Plastic Surgery Center, 2440 M Street NW, Suite 200, Washington, DC \\ 20037, USA
}

\section{ARTICLE INFO}

Received: 幽 February 17, 2021

Published: 幽 February 24, 2021

Citation: Paul G Ruff IV. Evaluation of Skin Tissue Effects of Two-Pass Helium Plasma Treatment in Comparison to Nitrogen Plasma Using a Swine Model. Biomed J Sci \& Tech Res 34(2)-2021. BJSTR. MS.ID.005512.

Keywords: Helium Plasma; Nitrogen Plasma; Skin Resurfacing; Porcine Model; Comparative Study

\section{ABSTRACT}

Background: Helium plasma is a new technology for dermal resurfacing. Objectives: To compare the depth of thermal effect produced by a two-pass skin treatment protocol for a helium plasma system in comparison to nitrogen plasma.

Methods: A 6-month-old domestic pig was anesthetized, intubated, and ventilated. Thirty-six $1.5 \mathrm{~cm} \times 1.5 \mathrm{~cm}$ square treatment sites were drawn on both shaved flanks. Energy was applied to each flank using helium and nitrogen plasma systems. Helium treatment was delivered as linear/non-overlapping strokes in single and double passes at $1 \mathrm{~cm} / \mathrm{sec}$ treatment speed. Helium plasma device settings were 20\% Power (energy density $8.6 \mathrm{~J} / \mathrm{cm}^{2}$ ) and $40 \%$ power (energy density $17.8 \mathrm{~J} / \mathrm{cm}^{2}$ ) with $4 \mathrm{lpm}$ of helium flow. Nitrogen treatment was delivered in consecutive pulses using the PSR3 treatment protocol ( $4.0 \mathrm{~J}, 2.5 \mathrm{~Hz}$ pulse rate and energy density $14.1 \mathrm{~J} / \mathrm{cm}^{2}$ ). Histology assessments of thermal injury were made from harvested tissue samples.

Results: The shallowest depth of thermal effect was $0.25 \pm 0.07 \mathrm{~mm}$ obtained using the helium plasma at $20 \%$ energy setting and a single pass. The deepest thermal effect was $0.72 \pm 0.07 \mathrm{~mm}$ obtained using the helium plasma at $40 \%$ energy setting with two passes. The maximum depth of thermal effect associated with the nitrogen plasma was $0.60 \pm 0.07$ mm and equivalent to helium plasma using $20 \%$ and $40 \%$ power with two passes.

Conclusions: There are no significant differences in the depth of thermal effect associated with a two-pass treatment protocol for the helium-based plasma system when compared to PSR3 treatment with a nitrogen-based plasma skin resurfacing system.

\section{Introduction}

The pursuit of smooth, youthful skin with even texture and tone is common in aesthetic practice. Numerous modalities through time have been used to achieve this end including chemical peeling, dermabrasion, lasers, radiofrequency and plasma sources. Each procedure has a unique interaction with the skin to achieve the desired result. Within the skin, the dermis contains well-organized collagen fibers that are responsible for the firmness and smoothness of the skin. As people age, collagen fibers become reduced in numbers and less organized, resulting in lax and wrinkled skin. In most skin resurfacing procedures, an energy source is used to selectively damage the skin, prompting a healing response that stimulates the growth of new, well-organized collagen fibers in the dermis resulting in smoother and firmer skin.
Physical plasma is an ionized gas, commonly referred to as the fourth state of matter to distinguish it from solids, liquids, and gases. Plasma is generated by applying a continuous supply of energy to a neutral gas until an excited state is achieved. The energy source needed to create plasma may be thermal, chemical or electrical in nature [1]. Based on its thermal properties, the role of plasma in medicine has expanded to include a wide range of applications including cancer [2], wound healing [3], infectious disease [4], and dentistry [5]. Plasma is also being used with increasing frequency in dermatology [6].

A nitrogen-based plasma device (NeoGen PSR System; Energist Medical Group formerly Rhytec, Swansea, United Kingdom) has 
FDA clearance for the treatment of dermatologic conditions including wrinkles and rhytides [7]. The system converts nitrogen gas into plasma energy within the tip of the handpiece which results in the expulsion of controlled pulses of energy to the tissue. The energy is not chromophore dependent and does not vaporize tissue $[8,9]$. The upper layers of the skin are desiccated during treatment and remain intact to serve as a biological dressing during the initial stages of healing $[8,9]$. The technology has demonstrated efficacy for the treatment of mild-to-moderate periorbital wrinkles and photodamaged facial skin with reported complications typical for resurfacing procedures such as postinflammatory hyperpigmentation, focal areas of delayed healing, and rare instances of hypertrophic scarring [8-13].

A helium-based plasma technology has FDA clearance for the cutting, coagulation, and ablation of soft tissue (Renuvion ${ }^{\circledR}$; $A$ pyx ${ }^{\mathrm{TM}}$ Medical Corporation, Clearwater, FL) [14]. This system consists of an electrosurgical generator, a handpiece, and a supply of helium gas. Radiofrequency (RF) energy is delivered to the handpiece by the generator and used to energize an electrode. When helium gas is passed over the energized electrode, a helium plasma is generated which allows for conduction of the RF energy from the electrode to the patient in the form of a precise, continuous (non-pulsed) helium plasma beam. Although initial clinical use has been as a general surgical tool in open and laparoscopic procedures, helium plasma has been recently evaluated for use in dermal resurfacing procedures. Holcomb and Schucker reported on the tissue effects of a low energy, single-pass helium plasma treatment in comparison to the nitrogen plasma system in a porcine model [15]. The results of this pre-clinical study were then translated into a prospective, 55-patient FDA investigational device exemption (IDE) clinical study in which subjects treated with a single pass from the helium device achieved significant improvements in facial appearance with rapid recovery and relatively few unanticipated adverse events [16]. Successful clinical use has also been reported outside of a controlled clinical study $[17,18]$.

The objective of this pre-clinical study was to evaluate the tissue effects of a second treatment pass of the helium plasma device using a naïve swine model. The tissue effects were compared to those of the established predicate nitrogen plasma device to support an assessment of the safety profile of two treatment passes prior to inclusion in additional clinical studies.

\section{Methods}

This study was performed in compliance with the US Public Health Service Policy on Humane Care and Use of Laboratory
Animals [19]. A 6-month-old female Yorkshire domestic cross swine was placed under general anesthesia, intubated, and mechanically ventilated. The hair from both lateral flanks was clipped. Thirty-six $1.5 \mathrm{~cm} \times 1.5 \mathrm{~cm}$ square treatment sites were drawn on the flanks with a permanent marker. The application of energy for both the helium plasma and nitrogen plasma systems was performed by the same user who had significant clinical experience with both devices.

Treatment delivery for the helium plasma system occurred as linear/non-overlapping strokes with both single pass and twopass protocols using a treatment speed of approximately $1 \mathrm{~cm} /$ sec. Although the primary focus of this study was the evaluation of the tissue effects caused by the two-pass protocol, the single pass treatment was included to allow for comparison to the data previously reported by Holcomb and Schucker [15]. Device settings for the helium plasma system consisted of 20\% Power (energy density $8.6 \mathrm{~J} / \mathrm{cm}^{2}$ ) and $40 \%$ power (energy density $17.8 \mathrm{~J} / \mathrm{cm}^{2}$ ) with $4 \mathrm{lpm}$ of helium flow. For the treatment protocols requiring two treatment passes, a saline soaked gauze was used to wipe the desiccated tissue between the first and second pass and the surface was dried of any moisture. Treatment using the nitrogen plasma system was performed using the highest energy (4.0), $2.5 \mathrm{~Hz}$ pulse rate and energy density $14.1 \mathrm{~J} / \mathrm{cm}^{2}$ ) double pass (PSR3) treatment protocol to establish the maximum treatment depth in clinical use for the device.

The spacer available for the system was used to ensure consistent distance of the treatment nozzle from the tissue, and proper spacing of the energy pulses was maintained to prevent overtreatment due to overlapping of treatment spots. Table 1 summarizes the treatment settings used for both devices. Six sites were treated for each of the five device settings tested, and six untreated sites were used as controls to provide a baseline for epidermal and dermal thicknesses for comparison when performing the histomorphometric evaluation.

Following treatment, skin tissue samples were harvested. After formalin fixation, tissue samples were cross-sectioned and embedded in paraffin blocks. Slides were cut and stained with hematoxylin \& eosin and Masson's trichrome stain per standard operating procedures. Images were obtained and thermal injury depth was measured using the Image-Pro image analysis software (Image-Pro Premier Software; Media Cybernetics $®$, Inc., Rockville, MD). The depth of thermal effect was measured to the deepest focus of dermal injury in three locations within the treated area (Figure 1). 

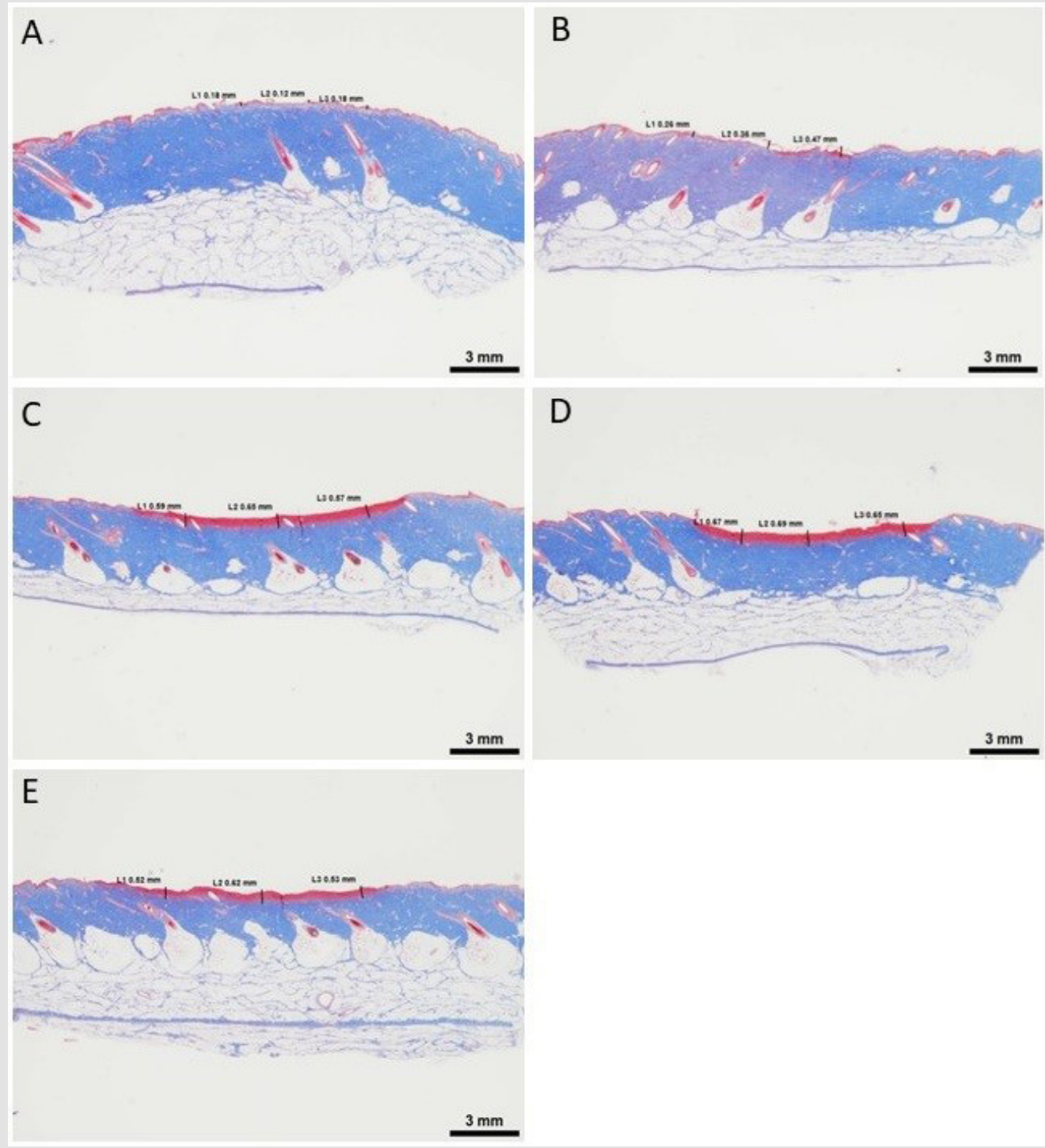

Figure 1: Depth of Thermal Effect

The depth of thermal effect was determined as the depth of the ablative and thermo-coagulation zones. Three depth measurements were made for each slide. Representative examples shown for (A) Helium Plasma 20\% Single Pass; (B) Helium Plasma 40\% Single Pass; (C) Helium Plasma 20\% Two-Pass; (D) Helium Plasma 40\% Two-Pass; and (E) Nitrogen Plasma PSR3.

Statistical analysis was conducted using Minitab (Version 14.13; Minitab, Inc, State College, PA). Statistical significance between different treatment conditions was determined by analysis of variance and Tukey's pairwise comparisons for groups.

\section{Results}

The maximum of the three measurements taken for each treatment location are reported in Table 1 . For the single pass helium plasma treatment, the $20 \%$ power setting resulted in an average maximum depth of $0.25 \pm 0.07 \mathrm{~mm}$, and the $40 \%$ power setting resulted in an average maximum depth of $0.43 \pm 0.11 \mathrm{~mm}$. These average maximum depth measurements for the single pass treatment are similar to those reported by Holcomb and Schucker of $0.22 \mathrm{~mm}$ for $20 \%$ power and $0.28 \mathrm{~mm}$ for $40 \%$ power [15]. The two-pass helium treatment protocol produced a deeper average maximum depth of $0.70 \pm 0.11 \mathrm{~mm}$ for $20 \%$ power and $0.72 \pm 0.11$ $\mathrm{mm}$ for $40 \%$ power. The nitrogen plasma PSR3 treatment recorded an average maximum depth of $0.60 \pm 0.07 \mathrm{~mm}$. To facilitate a comparison between the two-pass helium plasma treatment and the nitrogen PSR3 treatment, the average maximum depths were normalized against the nitrogen treatment and plotted in Figure 2. Increasing the number of passes of helium plasma treatment from one to two led to an increase in the maximum depth of thermal effect. Although there was a slight trend of higher maximum depth for both the $20 \%$ and $40 \%$ two-pass helium treatments when compared to the nitrogen plasma, the results were not statistically different. 
Table 1: Treatment Setting and Resulting Maximum Depth of Thermal Effect

\begin{tabular}{|c|c|c|c|c|}
\hline & Device Settings & Treatment Area & Max Depth (mm) & Mean Max Depth (mm) \\
\hline \multirow{24}{*}{ Helium Plasma System } & \multirow{6}{*}{$20 \%$ Power, 4 lpm, 1 pass } & 1 & 0.18 & \multirow{6}{*}{0.25} \\
\hline & & 2 & 0.22 & \\
\hline & & 3 & 0.34 & \\
\hline & & 4 & 0.3 & \\
\hline & & 5 & No Data & \\
\hline & & 6 & 0.19 & \\
\hline & \multirow{6}{*}{$40 \%$ Power, 4 lpm, 1 pass } & 1 & 0.4 & \multirow{6}{*}{0.43} \\
\hline & & 2 & 0.2 & \\
\hline & & 3 & 0.47 & \\
\hline & & 4 & 0.52 & \\
\hline & & 5 & 0.47 & \\
\hline & & 6 & 0.52 & \\
\hline & \multirow{6}{*}{$20 \%$ Power, 4 lpm, 2 passes } & 1 & 0.59 & \multirow{6}{*}{0.7} \\
\hline & & 2 & 0.64 & \\
\hline & & 3 & 0.62 & \\
\hline & & 4 & 0.9 & \\
\hline & & 5 & 0.65 & \\
\hline & & 6 & 0.77 & \\
\hline & \multirow{6}{*}{$40 \%$ Power, 4 lpm, 2 passes } & 1 & 0.67 & \multirow{6}{*}{0.72} \\
\hline & & 2 & 0.69 & \\
\hline & & 3 & 0.67 & \\
\hline & & 4 & 0.84 & \\
\hline & & 5 & 0.66 & \\
\hline & & 6 & 0.78 & \\
\hline \multirow{6}{*}{ Nitrogen Plasma System } & \multirow{6}{*}{ PSR3 } & 1 & 0.66 & \multirow{6}{*}{0.6} \\
\hline & & 2 & 0.67 & \\
\hline & & 3 & 0.62 & \\
\hline & & 4 & 0.47 & \\
\hline & & 5 & 0.57 & \\
\hline & & 6 & 0.61 & \\
\hline
\end{tabular}

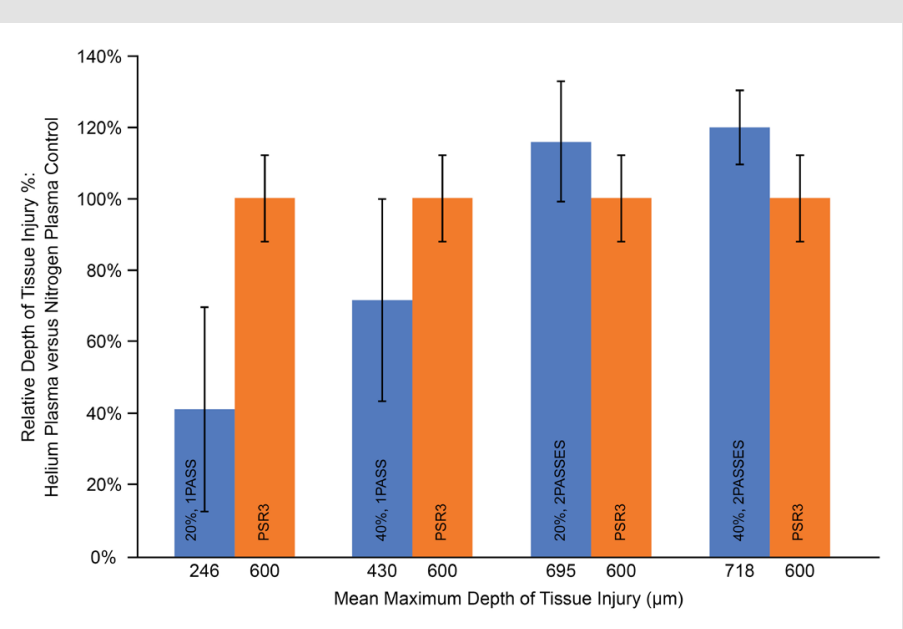

Figure 2: Acute Tissue Injury

The relative percent depth of acute tissue injury for helium plasma (blue) vs. nitrogen plasma control (orange). Treatment settings used and mean maximum depth of tissue injury also shown 


\section{Discussion}

For the past 30 years, multiple treatment modalities have been developed for dermal resurfacing for the purpose of treating facial wrinkles, dyschromias, and atrophic scars. Major advances over this time have made it possible to perform facial resurfacing safely with most adverse reactions being mild and easily treated. More severe complications such as hypertrophic scarring are rare but dreaded outcomes [20,21]. Although multiple factors can contribute to scar formation such as surgical technique, treatment of higher risk facial areas, and inadequate post-treatment wound management, one of the leading contributors is the depth of dermal injury [2022]. Therefore, when evaluating a new technology for the purpose of resurfacing, understanding the extent of thermal effect is a key element to establishing the safety profile of the device.

Nitrogen plasma was first introduced in 2006 as a new modality for resurfacing. The system offers multiple treatment protocols that remain a viable option for skin rejuvenation and have an acceptable safety profile. Recently, helium plasma has been studied in both pre-clinical and prospective clinical studies using a low energy, single pass treatment protocol $[15,16]$. The results of these studies indicated that the helium plasma device has the potential for effective, safe treatment of facial rhytidosis. Treated subjects achieved significant improvement in facial appearance, relatively few adverse events, and had high overall satisfaction with the procedure and aesthetic results [16].

The purpose of this study was to evaluate a more aggressive two-pass helium plasma treatment protocol prior to utilization in an FDA regulated clinical study. Since the nitrogen plasma device is most similar in method of action and has an established safety performance, it was chosen as a fitting predicate for comparison to the two-pass helium treatment in this study. With the primary focus being the safety of the new device setting, the depth of thermal injury was chosen as the primary endpoint. The data from this study shows that the depth of thermal effect of the two-pass helium plasma treatment is not statistically different from that produced by the most aggressive nitrogen PSR3 setting. Although this data does not by itself establish the safety of this new treatment protocol, combining this information with published histology results for other resurfacing technologies [23,24] and reports of clinical success using a two-pass helium treatment $[17,18]$ provides reasonable assurance that a two-pass treatment can transition safely to clinical study for certain skin types, facial regions, or clinical conditions. Less aggressive treatment should be considered for areas of thinner tissue such as the periorbital region or areas more prone to injury such as the mandibular border.

\section{Conclusion}

Helium-based plasma systems are relatively new in the aesthetic toolbox. Surface application of helium plasma energy may be a promising way to achieve dermal resurfacing. The results of this study indicated that there are not significant differences in the depth of thermal effect associated with a two-pass helium plasma treatment when compared to the depth of thermal effect associated with nitrogen-based plasma skin resurfacing systems. These results combined with other data provide reasonable assurance that a two-pass helium plasma treatment protocol can be used safely for certain skin types, facial regions, and/or clinical conditions. Further basic science and clinical research is warranted. A formal clinical study evaluating the safety and efficacy of the helium plasma device for dermal resurfacing is ongoing.

\section{Acknowledgement}

This study was sponsored by $\mathrm{Apyx}^{\mathrm{TM}}$ Medical Corporation, Clearwater, FL. The author acknowledges the editorial assistance of Dr. Carl S. Hornfeldt, Apothekon, Inc., during the preparation of this manuscript.

\section{References}

1. Von Woedtke T, Schmidt A, Bekeschus S, Kristian W, Klaus-Dieter W (2019) Plasma medicine: a field of applied redox biology. In Vivo 33(4): 1011-1026.

2. Yan D, Sherman JH, Keidar M (2017) Cold atmospheric plasma, a novel promising anti-cancer treatment modality. Oncotarget 8(9): 1597715995.

3. Kramer A, Conway BR, Meissner K, F Scholz, B H Rauch, et al. (2017) Cold atmospheric pressure plasma for treatment of chronic wounds: drug or medical device?. J Wound Care 26(8): 470-475.

4. Lis KA, Kehrenberg C, Boulaaba A, Blickwede MvK, Binder S, et al. (2018) Inactivation of multidrug-resistant pathogens and Yersinia enterocolitica with cold atmospheric-pressure plasma on stainless-steel surfaces. Int J Antimicrob Agents 52(6): 811-818.

5. Gherardi M, Tonini R, Colombo V (2018) Plasma in dentistry: brief history and current status. Trends Biotechnol 36(6): 583-585.

6. Gan L, Zhang S, Poorun D, Liu D, Lu X, et al. (2018) Medical applications of nonthermal atmospheric pressure plasma in dermatology. J Dtsch Dermatol Ges 16(1): 7-13.

7. (2020) Food and Drug Administration. 510(k) Clearance K060948.

8. Foster KW, Moy RL, Fincher EF (2008) Advances in plasma skin regeneration. J Cosmet Dermatol 7(3): 169-179.

9. Holcomb JD (2009) Nitrogen plasma skin regeneration and aesthetic facial surgery: Multicenter evaluation of concurrent treatment. Arch Facial Plast Surg 11(3): 184-193.

10. Theppornpitak N, Udompataikul M, Chalermchai T, Ophaswongse S, Limtanyakul P (2019) Nitrogen plasma skin regeneration for the treatment of mild-to-moderate periorbital wrinkles: a prospective, randomized, controlled evaluator-blinded trial. J Cosmet Dermatol 18: 163-168.

11. Bogle MA, Arndt KA, Dover JS (2007) Evaluation of plasma skin regeneration technology in low-energy full-facial rejuvenation. Arch Dermatol 143(2): 168-174.

12. Kilmer S, Semchyshyn N, Shah G, Fitzpatrick R (2007) A pilot study on the use of plasma skin regeneration device (Portrait PSR3) in full face rejuvenation procedures. Lasers Med Sci 22(2): 101-109.

13. Kono T, Groff WF, Sakuri H, Yamaki T, Soejima K, et al. (2009) Treatment of traumatic scars using plasma skin regeneration (PSR) system. Lasers Surg Med 41(2): 128-130.

14. (2020) Food and Drug Administration. 510k Clearance K170188. 
15. Holcomb JD, Schucker A (2019) Helium plasma skin regeneration: evaluation of skin tissue effects in a porcine model and comparison to nitrogen plasma skin regeneration. Lasers Surg Med 52(1): 23-32.

16. Holcomb JD, Kelly M, Hamilton TK, DeLozier JB (2020) A prospective study evaluating the use of helium plasma for dermal resurfacing. Lasers Surg Med 52(10): 940-951.

17. Gentile RD (2018) Cool atmospheric plasma (J-Plasma) and new options for facial contouring and skin rejuvenation of the heavy face and neck. Facial Plast Surg 34(1): 66-74.

18. Gentile RD (2019) Renuvion/J-Plasma for subdermal skin tightening facial contouring and skin rejuvenation of the face and neck. Facial Plast Surg Clin North Am 27(3): 273-290.

19. (2020) National Institutes of Health. Office of Laboratory Animal Welfare.

ISSN: 2574-1241

DOI: 10.26717/BJSTR.2021.34.005512

Paul G Ruff IV. Biomed J Sci \& Tech Res

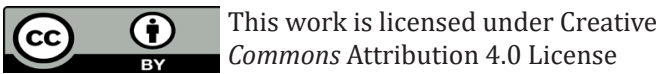

Submission Link: https://biomedres.us/submit-manuscript.php
20. Nanni CA, Alster TS (1998) Complications of carbon dioxide laser resurfacing. Dermatol Surg 24(3): 315-320.

21. Alster TS, Lupton JR (2000) Treatment of complications of laser skin resurfacing. Arch Facial Plast Surg 2(4): 279-284.

22. Dunkin CSJ, Pleat JM, Gillespie PH, Michael P H Tyler, Anthony $\mathrm{H}$ N Roberts, et al. (2007) Scarring occurs at a critical depth of skin injury: precise measurement in a graduated dermal scratch in human volunteers. Plast Reconstr Surg 119(6): 1722-1732.

23. Haina D, Landthaler M, Braun-Falco O, Waidelich W (1987) Comparison of the maximum coagulation depth in human skin for different types of medical lasers. Lasers Surg Med 7(4): 355-362.

24. Fitzpatrick R, Bernstein E, Iyer S, D Brown, P Andrews, et al. (2008) A histopathologic evaluation of the plasma skin regeneration system (PSR) versus a standard carbon dioxide resurfacing laser in an animal model. Lasers Surg Med 40(2): 93-99.

$\begin{array}{ll}\text { BIOMEDICAL } & \text { Assets of Publishing with us } \\ \text { RESEARCHES } & \text { - Global archiving of articles } \\ \text { - Immediate, unrestricted online access }\end{array}$

